\title{
Image-rotating cavity designs for improved beam quality in nanosecond optical parametric oscillators
}

\author{
A. V. Smith \\ Lasers, Optics and Remote Sensing Dept., Sandia National Laboratories, Albuquerque, NM 87185-1423 \\ email: arlsmit@sandia.gov \\ M. S. Bowers \\ Aculight Corp., 11805 North Creek Pkwy S. Suite 113, Bothell, WA 98011
}

\begin{abstract}
We show by computer simulation that high beam quality can be achieved in high energy, nanosecond optical parametric oscillators by using image-rotating resonators.

OCIS: 190.4970 Parametric oscillators and amplifiers, 190.4420 Nonlinear optics, transverse effects in

One of the greatest challenges in scaling nanosecond optical parametric oscillators (OPO's) to high energy is obtaining good beam quality along with high efficiency. Because of constraints imposed by crystal nonlinearities and damage thresholds, scaling an OPO from low to high energy implies increasing the beam diameters so the fluences, crystal lengths, and cavity length can remain relatively unchanged. The result is a high-Fresnel-number cavity that can support many transverse modes, often resulting in poor beam quality. We have applied numerical models to examine the effectiveness of various image-rotating resonator designs in improving signal and idler beam quality for high energy, nanosecond OPO's. The numerical model for monochromatic OPO's was described in an earlier paper ${ }^{1}$. We demonstrated that this model accurately predicted beam quality for a low-Fresnel-number, three-mirror-ring, KTP OPO for pump levels 2.5 times threshold and $\mathrm{M}^{2}$ ss of 4 . The model includes diffraction and birefringent walk off but not group velocity effects. The input pump and signal waves are assumed to be perfect spatial Gaussian beams. We believe the fact that the initial signal beam is a perfect Gaussian does not invalidate the model for the purpose of beam quality studies because in the amplification process the beam is greatly gain narrowed due to the nonuniform pump profile. The signal wave reaches threshold first at the center of the pump beam, so at the time of turn on it has a small diameter and thus populates many transverse plane-wave components. Additionally, after turn on back conversion distorts the signal and idler waves, and this also populates high order transverse components. Beam clean up must thus occur after turn on so the starting profile is relatively unimportant.
\end{abstract}

Image rotating cavities have long been used to improve the beam quality of lasers ${ }^{2}$. On successive passes each portion of the laser beam samples different gain regions, averaging to some extent the gain and refractive index inhomogeneities. This mechanism can also be effective in OPO's where inhomogeneities of the pump light can be averaged. However, the principal clean-up mechanism in OPO's is not gain averaging but the establishment of phase and amplitude correlations across the signal and idler beams due to lateral walk off between them as they propagate through the crystal. For type II mixing, the birefringent walk off in a single pass of a critically phase matched crystal is typically $0.1-1 \mathrm{~mm}$. This can be a significant fraction of the beam diameter. By its nature, parametric gain tends to establish local phase and amplitude correlations between the signal and idler beams, and also within the signal beam and within the idler beam, over stripes of length equal to the walk off, and oriented parallel to walk off. Over multiple passes through the OPO cavity these stripes can grow to cover the full signal and idler beam widths. A consequence of this correlation is that the beam divergence in the walk off direction can be restricted to near the diffraction limit, while the divergence in the other direction is less restricted. This effect is often somewhat obscurely referred to as a restricted crystal acceptance angle and it is usually analyzed in terms of tilted plane waves. The analysis presented here in terms of correlation zones is equivalent to tilted plane waves for monochromatic operation but is easier to apply and visualize ${ }^{3,4}$.

An example of divergence asymmetry may be seen in the figures below. The first figure shows the OPO which has a single crystal with walk off between signal and idler in the $x$-direction as indicated by the arrow on the crystal. The ring cavity lies in the $x$ - $z$ plane and inverts the resonated signal beam in the $x$ dimension on each pass. The idler and pump are not resonated. The Fresnel number is about 35 here and the OPO is pumped about twice threshold. 


\section{DISCLAIMER}

This report was prepared as an account of work sponsored by an agency of the United States Government. Neither the United States Government nor any agency thereof, nor any of their employees, make any warranty, express or implied, or assumes any legal liability or responsibility for the accuracy, completeness, or usefulness of any information, apparatus, product, or process disclosed, or represents that its use would not infringe privately owned rights. Reference herein to any specific commercial product, process, or service by trade name, trademark, manufacturer, or otherwise does not necessarily constitute or imply its endorsement, recommendation, or favoring by the United States Government or any agency thereof. The views and opinions of authors expressed herein do not necessarily state or reflect those of the United States Government or any agency thereof. 


\section{DISCLAIMER}

Portions of this document may be illegible in electronic image products. Images are produced from the best available original document. 


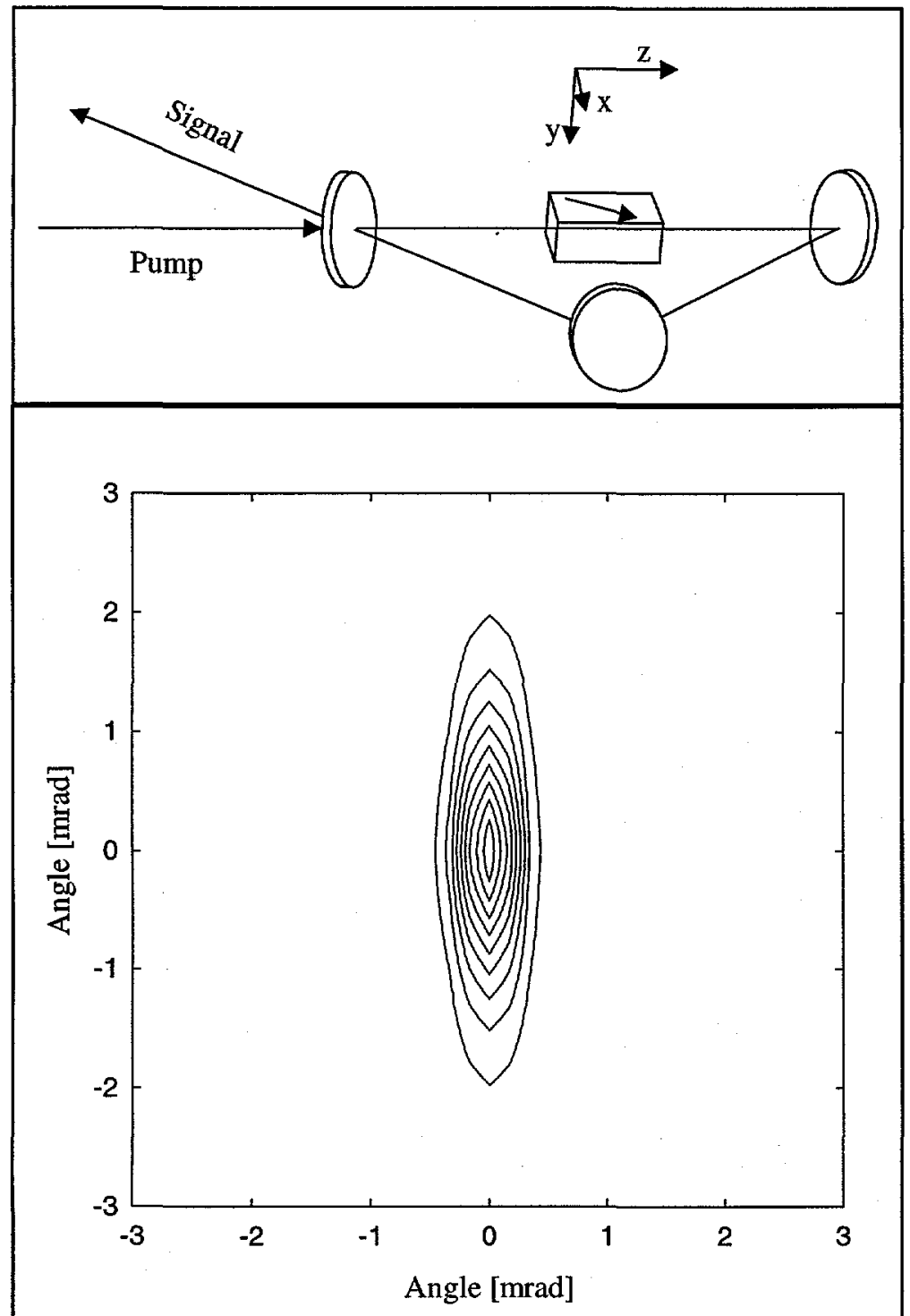

In the lower figure we show a model-generated far-field signal fluence for this OPO. As expected, correlations in the signal beam develop over many passes as stripes in the $x$-direction (horizontal axis), leading to reduced divergence in that direction. Correlation between stripes is weak, however, because diffractive coupling is weak in the $y$-direction. This decoupling length is considerably less than the pump beam diameter, so the divergence in the $y$-direction (vertical axis) is relatively large.

If the image of the resonated signal wave is rotated $90^{\circ}$ on each cavity pass, the correlation zone can extend in both transverse dimensions, leading to improved beam quality in both dimensions. The figure below shows one example of an imagerotating cavity, along with a diagram of the correlation zones for successive passes. A dove prism in the return leg, rotated $45^{\circ}$ relative to the ring plane, rotates the signal beam by $90^{\circ}$ on each pass. Waveplates before and after the prism align the signal beam polarization to an eigenpolarization of the dove prism to avoid depolarization. In

the diagrams of the correlation zones we show how the signal field initially at the origin develops zones connected by idler walk off and image rotation. The open circle indicates the origin. After the first pass through the crystal, a correlation zone is created that extends in the $x$, or walk off direction, a distance equal to the single pass idler walk off, $\rho L_{\text {crystal }}$. Here $\rho$ is the angle between signal and idler Poynting vectors in the crystal, whether due to
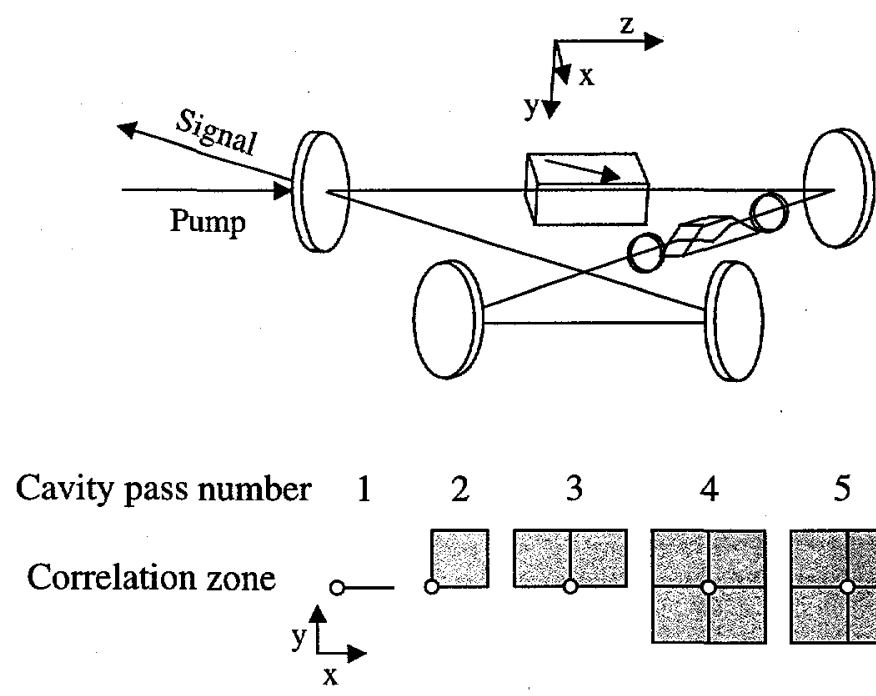

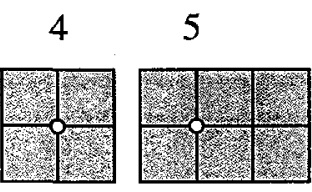


birefringence or noncollinear pumping. This is shown under pass number 1. This line is rotated counter clockwise by $90^{\circ}$ in the dove prism so that on the next pass through the crystal the walk off again creates correlations in the $x$ direction, as shown under pass number 2. Repetition of the counter clockwise rotation plus walk off on successive crystal passes creates the growing correlation zones diagramed. In the last figure we display the nearly symmetric far-field signal fluence computed for this device. The effectiveness of image rotation is apparent in both the far-field fluence pattern and in the low values for the signal $\mathrm{M}^{2}$ in both directions.

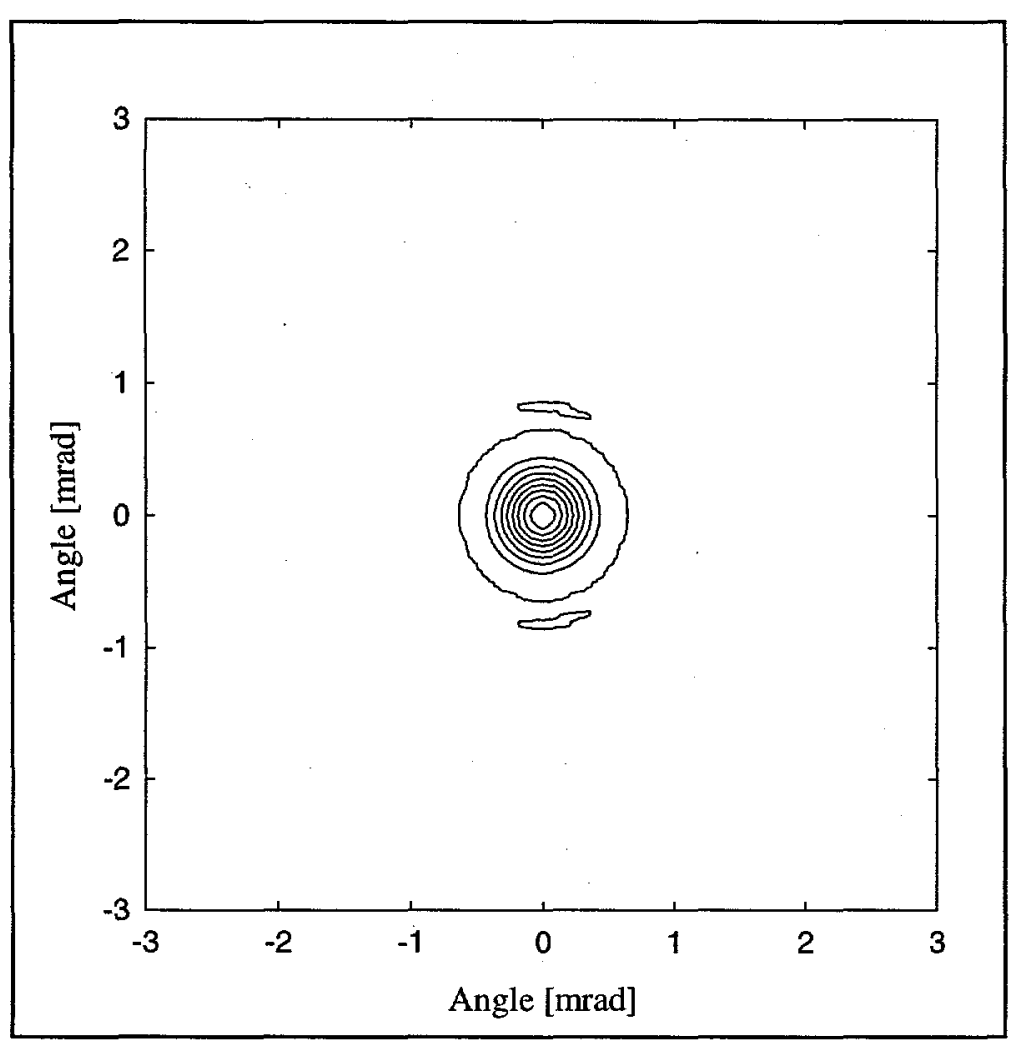

This design with $90^{\circ}$ image rotation is one of many designs that induce correlations across the full signal and idler beams. We have studied several others as well and find that if the correlation zones develop over the full signal beam the beam quality is dramatically improved compared with designs that induce asymmetric or onedimensional correlation zones. We have not yet demonstrated this in the laboratory but we can point to the design of Nabors and Frangineas ${ }^{4}$ that creates symmetric correlation zones by using noncollinear pumping in a type I, BBO OPO with a prism beam inverter. They measure improved beam quality relative to a similar collinearly pumped $O P O$ that does not develop correlation zones. We will show alternate designs and indicate their predicted performance based on the our numerical model.

We believe the monochromatic models applied in this study should apply to both monochromatic and broadband OPO's, although this has not been validated. In broad bandwidth OPO's there is temporal as well as spatial walk off so the development of correlation zones is more complicated than for the monochromatic case. Three dimensional zones must be considered rather than two dimensional. From numerous experimental studies of OPO linewidth, we know that the temporal extent of the walk off is about equal to the single-pass temporal walk off between signal and idler multiplied by the number of passes during the pulse. This implies that over this temporal zone the transverse correlations can still develop, so our model should still be quite good. Limited comparison between a broad-bandwidth model ${ }^{3}$ and the monochromatic model supports this conclusion.

References:

1. A. V. Smith, W. J. Alford, T. D. Raymond, and M. S. Bowers, "Comparison of a numerical model with measured performance of a seeded nanosecond KTP optical parametric oscillator," J. Opt. Soc. Am. B 12, 2253-2267 (1995).

2. Y. A. Anan'ev, Laser resonators and beam divergence problem, Adam Hilger, New York 1992, pp 321-327.

3. W. J. Alford, R. J. Gehr, R. L. Schmitt, A. V. Smith, and G. Arisholm, "Beam tilt and angular dispersion in broad-bandwidth, nanosecond optical parametric oscillators," J. Opt. Soc. Am. B 16, 1525-1532 (1999).

4. A. V. Smith, D. J. Armstrong, and W. J. Alford, "Increased acceptance bandwidths in optical frequency conversion by use of multiple walk-off-compensating nonlinear crystals," J. Opt. Soc. Am. B 15, 122-141 (1998).

5. C. D. Nabors and G. Frangineas, "Optical parametric oscillator with bi-noncollinear, porro prism cavity," OSA TOPS \{lbf 10,\} Advanced Solid State Lasers, 1997, C. R. Pollock and W. R. Bosenberg (eds.) 90-93 (1997). C. D. Nabors and G. Frangineas, US Patent No. 5,781,571.

Acknowledgement: This work was supported by the United States Department of Energy under contract DE-AC04-94AL85000. Sandia is a multiprogram laboratory operated by Sandia Corporation, a Lockheed Martin Company, for the United States Department of Energy. 